\title{
Dynamics of Rumor Spreading in Complex Networks
}

\author{
Yamir Moreno, ${ }^{1,2}$ Maziar Nekovee, ${ }^{3}$ and Amalio F. Pacheco ${ }^{1,2}$ \\ ${ }^{1}$ Departamento de Física Teórica, Universidad de Zaragoza, Zaragoza 50009, Spain \\ ${ }^{2}$ Instituto de Biocomputación y Física de Sistemas Complejos, \\ Universidad de Zaragoza, Zaragoza 50009, Spain \\ ${ }^{3}$ Complexity Research Group, Polaris 134 BT Exact, Martlesham, Suffolk IP5 3RE, UK
}

(Dated: February 2, 2008)

\begin{abstract}
We derive the mean-field equations characterizing the dynamics of a rumor process that takes place on top of complex heterogeneous networks. These equations are solved numerically by means of a stochastic approach. First, we present analytical and Monte Carlo calculations for homogeneous networks and compare the results with those obtained by the numerical method. Then, we study the spreading process in detail for random scale-free networks. The time profiles for several quantities are numerically computed, which allow us to distinguish among different variants of rumor spreading algorithms. Our conclusions are directed to possible applications in replicated database maintenance, peer to peer communication networks and social spreading phenomena.
\end{abstract}

PACS numbers: 89.75.-k, 89.75.Fb, 05.70.Jk, 05.40.a

\section{INTRODUCTION}

During the last years, many systems have been analyzed from the perspective of graph theory [1, 2]. It turns out that seemingly diverse systems such as the Internet, the World Wide Web (WWW), metabolic and protein interaction networks and food webs, to mention a few examples, share many topological properties [3]. Among these properties, the fact that one can go from one node (or element) of the network to another node passing by just a few others is perhaps the most popular property, known as "six degrees of separation" or small-world (SW) property $[3,4]$. The SW feature has been shown to improve the performance of many dynamical processes as compared to regular lattices; a direct consequence of the existence of key shortcuts that speed up the communication between otherwise distant nodes and of the shorter path length among any two nodes on the net [1, 2, 3].

However, it has also been recognized that there are at least two types of networks fulfilling the SW property but radically different as soon as dynamical processes are ran on top of them. The first type can be called "exponential networks" since the probability of finding a node with connectivity (or degree) $k$ different from the average connectivity $\langle k\rangle$ decays exponentially fast for large $k$ [5]. The second kind of networks comprises those referred to as "scale-free" (SF) networks [ 6 ]. For these networks, the probability that a given node is connected to $k$ other nodes follows a power-law of the form $P(k) \sim k^{-\gamma}$, with the remarkable feature that $\gamma \leq 3$ for most real-world networks [1, 2].

The heterogeneity of the connectivity distribution in scale-free networks greatly impacts the dynamics of processes that they support. One of the most remarkable examples is that an epidemic disease will pervade in an infinite-size SF network regardless of its spreading rate [7, 8, 9, 10, 11]. The change in the behavior of the processes is so radical in this case that it has been claimed that the standard epidemiological framework should be carefully revisited. This might be bad news for epidemiologists, and those fighting natural and computer viruses. On the other hand, in a number of important technological and commercial applications, it is desirable to spread the "epidemic" as fast and as efficient as possible, not to prevent it from spreading. Important examples of such applications are epidemic (or rumor-based) protocols for data dissemination and resource discovery on the Internet [17, 18, 19, 20], and marketing campaigns using rumorlike strategies (viral marketing).

The above applications, and their dynamics, have passed almost unnoticed 22, 23] to the physics community working on complex networks despite the fact that they have been extensively studied by computer scientists and sociologists [20, 24]. The problem here consists of designing an epidemic (or rumor-mongering) algorithm in such a way that the dissemination of data or information from any node of a network reaches the largest possible number of remaining nodes. Note that in this case, in contrast to epidemic modeling, one is free to design the rules of epidemic infection in order to reach the desired result, instead of having to model an existing process. Furthermore, in a number of applications, such as peer-to-peer file sharing systems [19] built on top of the Internet and grid computing 21, the connectivity distribution of the nodes can also be changed in order to maximize the performance of such protocols.

In this paper we study in detail the dynamics of a generic rumor model [25] on complex scale-free topologies through analytic and numerical studies, and investigate the impact of the interaction rules on the efficiency and reliability of the rumor process. We first solve the model analytically for the case of exponential networks in the infinite time limit and then introduce a stochastic approach to deal with the numerical solution of the mean-field rate equations characterizing the system's dynamics. The method [26, 27, 28] is used to obtain accurate results for several quantities when the topology of random SF networks is taken into account, without us- 
ing large and expensive Monte Carlo (MC) simulations. The rest of the paper is organized as follows. Section II is devoted to introducing the rumor model and to derive the mean-field rate equations used throughout the paper. In Section III we deal with the stochastic approach, and compare its performance with analytical and MC calculations in homogeneous systems. We extend the method to the case of power-law distributed networks and present the results obtained for this kind of networks in Section IV and V. Finally, the paper is rounded off in the last Section, where conclusions are given.

\section{RUMOR MODEL IN HOMOGENEOUS NETWORKS}

The rumor model is defined as follows. Each of the $N$ elements of the network can be in three different states. Following the original terminology and the epidemiological literature 24], these three classes correspond to ignorant, spreader and stifler nodes. Ignorants are those individuals who have not heard the rumor and hence they are susceptible to be informed. The second class comprises active individuals that are spreading the rumor. Finally, stiflers are those who know the rumor but that are no longer spreading it. The spreading process evolves by directed contacts of the spreaders with others in the population. When a spreader meets an ignorant the last one turns into a new spreader with probability $\lambda$. The decay of the spreading process may be due to a mechanism of "forgetting" or because spreaders learn that the rumor has lost its "news value". We assume this latter hypothesis as the most plausible so that the contacting spreaders become stiflers with probability $\alpha$ if they encounter another spreader or a stifler. Note that as we are designing our rumor strategy in such a way that the fraction of the population which ultimately learns the rumor be the maximum possible, we have assumed that contacts of the type spreader-spreader are directed, that is, only the contacting individual loses the interest in propagating the rumor further. Therefore, there is no double transition to the stifler class.

In a homogeneous system, the original rumor model due to Daley and Kendall [25] can be described in terms of the densities of ignorants, spreaders, and stiflers, $i(t)$, $s(t)$, and $r(t)$, respectively, as a function of time. Besides, we have the normalization condition,

$$
i(t)+s(t)+r(t)=1 .
$$

In order to obtain an analytical insight and a way to later test our numerical approach, we first study the rumor model on top of exponentially distributed networks. These include models of random graphs as well as the Watts and Strogatz (WS) small-world model [3, 4]. This model produces a network made up of $N$ nodes with at least $m$ links to other nodes. The resulting connectivity distribution in the random graph limit of the model [4] takes the form

$$
P(k)=\frac{m^{k-m}}{(k-m) !} e^{-m},
$$

which gives an average connectivity $\langle k\rangle=2 m$. Hence, the probability that a node has a degree $k \gg\langle k\rangle$ decays exponentially fast and the network can be regarded as homogeneous.

The mean-field rate equations for the evolution of the three densities satisfy the following set of coupled differential equations:

$$
\begin{aligned}
& \frac{d i(t)}{d t}=-\lambda\langle k\rangle i(t) s(t), \\
& \frac{d s(t)}{d t}=\lambda\langle k\rangle i(t) s(t)-\alpha\langle k\rangle s(t)[s(t)+r(t)], \\
& \frac{d r(t)}{d t}=\alpha\langle k\rangle s(t)[s(t)+r(t)],
\end{aligned}
$$

with the initial conditions $i(0)=(N-1) / N, s(0)=$ $1 / N$ and $r(0)=0$. The above equations state that the density of spreaders increases at a rate proportional to the spreading rate $\lambda$, the average number of contacts of each individual $\langle k\rangle$ and to the densities of ignorant and spreader individuals, $i(t)$ and $s(t)$, respectively. On the other hand, the annihilation mechanism considers that spreaders decay into the stifler class at a rate $\alpha\langle k\rangle$ times the density of spreaders and of non-ignorant individuals $1-i(t)=s(t)+r(t)$.

The system of differential equations (24) can be analytically solved in the infinite time limit when $s(\infty)=0$. Using equation (1), we have that $\int_{0}^{\infty} s(t) d t=r_{\infty}=$ $\lim _{t \rightarrow \infty} r(t)$. Introducing the new variable $\beta=1+\lambda / \alpha$ we obtain the transcendental equation,

$$
r_{\infty}=1-e^{-\beta r_{\infty}} .
$$

Equation (57) always admits the trivial solution $r_{\infty}=$ 0 , but at the same time it also has another physically relevant solution for all values of the parameters $\lambda$ and $\alpha$. This can be easily appreciated since the condition,

$$
\left.\frac{d}{d r_{\infty}}\left(1-e^{-\beta r_{\infty}}\right)\right|_{r_{\infty}=0}>1
$$

reduces to $\lambda / \alpha>0$. That is, there is no "rumor threshold" contrary to the case of epidemic spreading [8]. This strikingly different behavior does not come from any difference in the growth mechanism of $s(t)$-the two are actually the same-, but from the disparate rules for the decay of the spreading process.

On the other hand, this result also points out that a mathematical model for the spreading of rumors can be constructed in many different ways. The results of this paper, however, indicate that the presence of spreader annihilation terms due to spreader-spreader and spreaderstifler interactions is very relevant for practical implementations 29, 30]. We shall come back to this point later on. 


\begin{tabular}{|c|c|r|r|}
\hline \hline$\alpha$ & Eq. (5) & MC & SNA \\
\hline 1 & 0.7968 & 0.813 & 0.802 \\
0.5 & 0.9404 & 0.962 & 0.954 \\
0.25 & 0.9930 & 0.986 & 0.987 \\
0.2 & 0.9974 & 0.996 & 0.997 \\
0.1 & 0.9999 & 0.998 & 0.999 \\
\hline \hline \hline
\end{tabular}

TABLE I: Density of stiflers at the end of the rumor spreading process. Results are shown for 5 different values of $\alpha$ for each method considered. Monte Carlo (MC) simulations were performed in a WS network with $\langle k\rangle=6$ and $N=10^{4}$ nodes. The same system size was used in the stochastic numerical approach (SNA).

\section{STOCHASTIC NUMERICAL APPROACH}

Recently 28], we have introduced a numerical technique [26] to deal with the mean-field rate equations appearing in epidemic-like models. It solves the differential equations by calculating the passage probabilities for the different transitions. The main advantage of this method, as compared to MC simulations, is its modest memory and CPU time requirements for large system sizes. Besides, we do not have to generate any network. Instead, we produce a sequence of integers distributed according to the desired connectivity distribution $P(k)$. The numerical procedure here proceeds as follows. At each time step until the end of the rumor spreading process, the following steps are performed:

1. Identify from the mean-field rate equations the transition probabilities per time unit from one state into the following one, that is, from the $i$ class to the $s$ class, $W_{i \rightarrow s}$, and finally to the $r$ class, $W_{s \rightarrow r}$.

2. Calculate the mean time interval, $\tau$, for one transition to occur. This is determined as the inverse of the sum of all the transition probabilities; $\tau=1 /\left(W_{i \rightarrow s}+W_{s \rightarrow r}\right)$.

3. Stochastically decide what transition will actually take place. This is done by deciding that the probabilities for both transitions are given by $\Pi_{i \rightarrow s}=$ $W_{i \rightarrow s} \tau$ and $\Pi_{s \rightarrow r}=W_{s \rightarrow r} \tau$, respectively, materializing the choice by generating a random number between 0 and 1 .

The numerical algorithm described above does not depend on the topological features of the network on top of which the rumor dynamics is taking place. Indeed, all the topological information, including correlations, enters in the computation of the transition probabilities. We should note here that the present results are obtained for uncorrelated networks. The method could also be applied to correlated networks without explicit generation of them. In that case, one should work with the two point correlation function $P\left(k, k^{\prime}\right)$ [28] instead of using $P(k)$.
On the other hand, a correlated network could be built up as in 31].

In order to gain confidence with the method and to show its soundness, we show in Table $\square$ the values of $r_{\infty}$ obtained from Eq. (5), MC simulations and the stochastic approach for homogeneous networks. In this case, the transition probabilities are the same for all the elements within a given class $(i, s$ or $r)$ irrespective of their actual connectivities. From equations (24) we get

$$
\begin{aligned}
W_{i \rightarrow s}(t) & =N \lambda\langle k\rangle i(t) s(t), \\
W_{s \rightarrow r}(t) & =N \alpha\langle k\rangle s(t)[s(t)+r(t)],
\end{aligned}
$$

for the transitions from the ignorant to the spreader class and from the spreader to the stifler class, respectively.

It can be seen from Table \that the difference between the SNA result and the MC simulations is less that 1.4\%, indicating the reliability of the SNA approach. The remaining small differences between the SNA and the MC results is mainly due to the fact that the homogeneous SNA model does not take into account the exponentially decaying fluctations in the connectivity of WS networks. On the other hand, MC simulations of the rumor dynamics for a network made up of $N=10^{4}$ nodes, averaged over at least 10 different network realizations and 1000 iterations, took several hours. Eventually, this method takes up to a few days when increasing the system size and decreasing the value of $\alpha$. On the contrary, the stochastic approach is very fast. Indeed, for the same parameter values, the numerical simulation takes around 5 minutes CPU time in a 2.0Ghz-P4 PC. Therefore, having such a method will allow us to scrutinize very efficiently and accurately the whole phase diagram and time profiles of the process under study. In what follows, we analyze in detail the dynamics of the rumor spreading process by numerically solving the mean-field rate equations for $\mathrm{SF}$ networks.

\section{POWER-LAW DISTRIBUTED NETWORKS}

The heterogeneity of the connectivity distribution inherent to SF networks significantly affects the dynamical evolution of processes that take place on top of these networks $7,8,19,10,11,12,13,14,32$. We have learned in recent years that the fluctuations of the connectivity distribution, $\left\langle k^{2}\right\rangle$, can not be neglected even for finite size systems [2]. Thus, the system of differential equations (24) should be modified accordingly. In particular, we should take into account that nodes could not only be in three different states, but also they belong to different connectivity classes $k$. Let us denote by $i_{k}(t), s_{k}(t)$, and $r_{k}(t)$ the densities of ignorants, spreaders and stiflers with connectivity $k$, respectively. In addition, we have that $i_{k}(t)+s_{k}(t)+r_{k}(t)=1$. The mean-field rate 
equations now read as,

$$
\begin{aligned}
\frac{d i_{k}(t)}{d t}= & -\lambda k i_{k}(t) \sum_{k^{\prime}} \frac{k^{\prime} P\left(k^{\prime}\right) s_{k^{\prime}}(t)}{\langle k\rangle} \\
\frac{d s_{k}(t)}{d t}= & \lambda k i_{k}(t) \sum_{k^{\prime}} \frac{k^{\prime} P\left(k^{\prime}\right) s_{k^{\prime}}(t)}{\langle k\rangle} \\
& -\alpha k s_{k}(t) \sum_{k^{\prime}} \frac{k^{\prime} P\left(k^{\prime}\right)\left[s_{k^{\prime}}(t)+r_{k^{\prime}}(t)\right]}{\langle k\rangle} \\
\frac{d r_{k}(t)}{d t}= & \alpha k s_{k}(t) \sum_{k^{\prime}} \frac{k^{\prime} P\left(k^{\prime}\right)\left[s_{k^{\prime}}(t)+r_{k^{\prime}}(t)\right]}{\langle k\rangle}
\end{aligned}
$$

where $P(k)$ is the connectivity distribution of the nodes and $\sum_{k^{\prime}} k^{\prime} P\left(k^{\prime}\right) s_{k^{\prime}}(t) /\langle k\rangle$ is the probability that any given node points to a spreader. We start from a randomly selected spreader and all the remaining nodes in the ignorant class. The summation in Eq. (10) stands for the probability that a node points to a spreader or a stifler. Note that as before, we do not allow for double transitions from the spreader to the stifler class. Next, we compute the respective transition probabilities. In this case, we should also consider that transitions from one state into another also take place within connectivity classes. Thus, the transition probabilities depend on $k$ as well. From Eq. (10) we obtain,

$$
\begin{aligned}
& W_{i \rightarrow s}(t, k)=\lambda k N P(k) i_{k}(t) \sum_{k^{\prime}} \frac{k^{\prime} P\left(k^{\prime}\right) s_{k^{\prime}}(t)}{\langle k\rangle}, \\
& W_{s \rightarrow r}(t, k)=\alpha k N P(k) s_{k}(t) \sum_{k^{\prime}} \frac{k^{\prime} P\left(k^{\prime}\right)\left[s_{k^{\prime}}(t)+r_{k^{\prime}}(t)\right]}{\langle k\rangle}
\end{aligned}
$$

where all the topological information is contained. Finally for the mean time interval after $i-1$ transitions, $\tau$, we find at each time step

$$
\tau=\frac{1}{W_{i \rightarrow s}(t)+W_{s \rightarrow r}(t)},
$$

with $W_{i \rightarrow s}(t)=\sum_{k} W_{i \rightarrow s}(t, k), \quad W_{s \rightarrow r}(t)=$ $\sum_{k} W_{s \rightarrow r}(t, k)$ and $t=\sum_{j}^{i-1} \tau_{j}$, where the $\tau_{j}$ s are the mean times of the $i-1$ previous transitions. At this point, the identification of what transition takes place and which connectivity class is affected proceeds as defined in step 3 of the previous section.

\section{RESULTS AND DISCUSSION}

The stochastic method described above can be used to explore several quantities characterizing the dynamics of the rumor spreading process. Throughout the rest of the paper we set $\lambda=1$ without loss of generality and vary the value of $\alpha$. We first generated a sequence of integers distributed according to $P(k) \sim k^{-\gamma}$ with $\gamma=3$ and

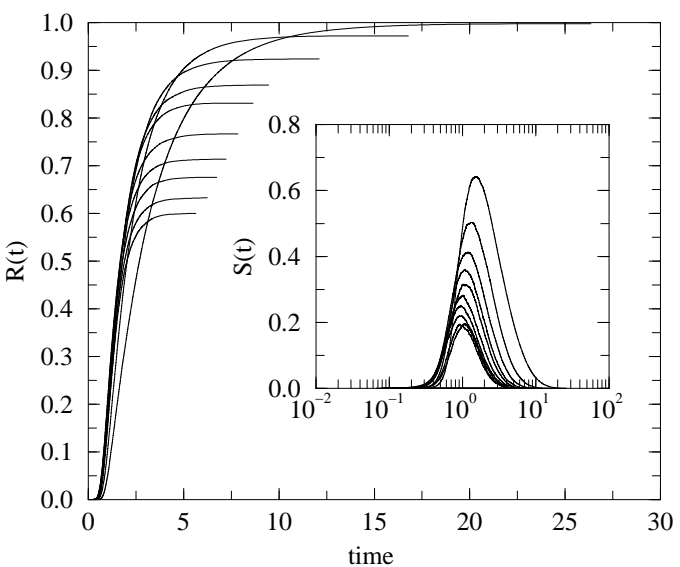

FIG. 1: Time evolution of the density of stifler individuals for different values of $\alpha$. From below, the values of $\alpha$ go from 1.0 to 0.1 at fixed increments of 0.1 . The inset shows the time dependency of the density of spreaders. The system size is $N=10^{4},\langle k\rangle=6$ and $\gamma=3$. Time is in units of $\alpha^{-1}$.

$\langle k\rangle=6$. As initial condition we use $r_{k}(t=0)=0$, and

$$
s_{k}(t)= \begin{cases}\frac{1}{N P(k)} & k=k_{i} \\ 0 & \text { otherwise }\end{cases}
$$

where $k_{i}$ is the connectivity of the randomly chosen initial spreader. The results are then averaged over at least 1000 different choices of $k_{i}$.

One of the most important practical aspects of any rumor mongering process is whether or not it reaches a high number of individuals. This magnitude is simply given by the final density of stiflers and is called reliability of the rumor process. However, it is also of great importance for potential applications that higher levels of reliability are reached as fast as possible, which constitute a practical measure of the cost associated to such levels of stiflers. For example, in technological applications, where one may consider several strategies [29, 30], it is possible to define a key global quantity, the efficiency of the process, which is the ratio between the reliability and the traffic imposed to the network. For these applications it is not only important to have high levels of reliability but also to achieve these with the lowest possible load resulting from the epidemic protocol's message passing traffic. This is important in order to avoid network congestion and also to reduce the amount of processing power used by nodes participating in the rumor process.

In order to analyze, from a global perspective, this trade-off between reliability and cost, we use time as a practical measure of efficiency. We call a rumor process less efficient than another if it needs more time to reach the same level of reliability. Figure 1 shows the time evolution of the density of stiflers for several values of the parameter $\alpha$. It turns out, as expected, that the number of individuals who finally learned the rumor increases as the probability of becoming stifler decreases. On the other 


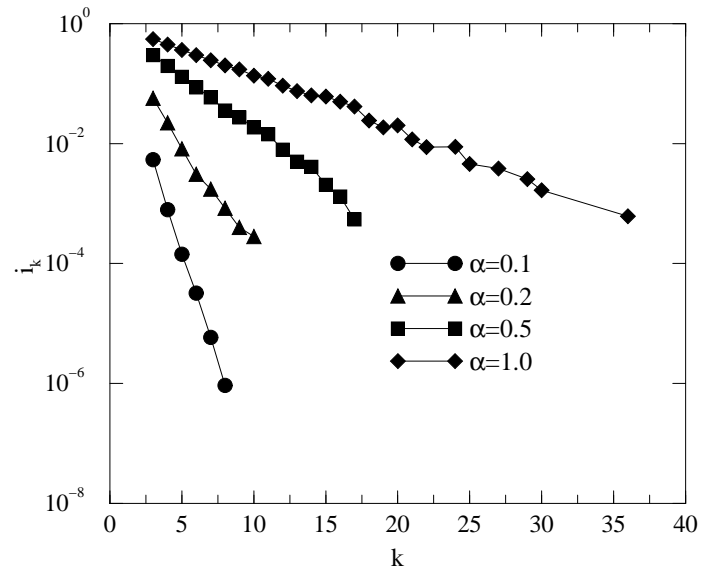

FIG. 2: Density of ignorants $i_{k}$ at the end of the rumor process as a function of their connectivity $k$. A clear exponential decay can be appreciated for all values of $\alpha$ shown. This implies that hubs have efficiently learned the rumor.

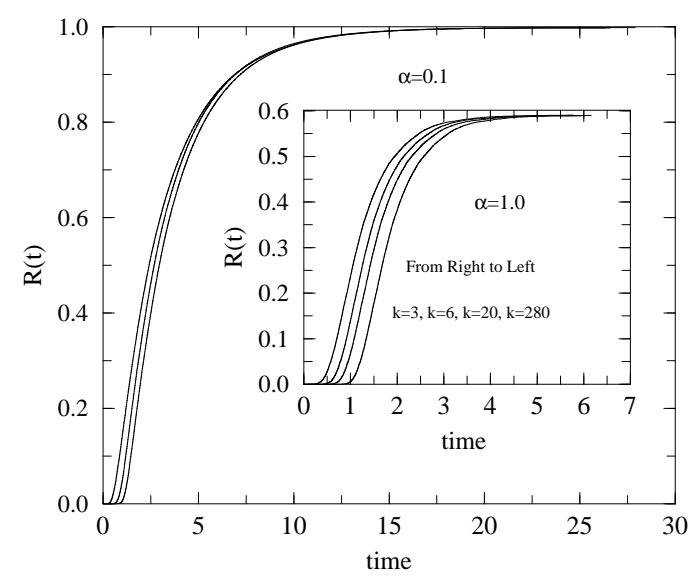

FIG. 3: Density of stiflers as a function of time for $\alpha=0.1$ (main figure) and $\alpha=1.0$ (inset) when the initial spreader has the connectivity indicated in the inset. Note that in all cases the final density of individuals who have learned the rumor is the same, but the asymptotic value is reached at different times. The model parameters are as of Fig. (1). Time is in units of $\alpha^{-1}$.

hand, the time it takes for $R(t)$ to reach its asymptotic value slightly increases with $\alpha^{-1}$, but clear differences do not arise for the two extreme values of $\alpha$. In fact, for a given time after the beginning of the rumor propagation, the density of stiflers scales with the inverse of $\alpha$. This behavior is further corroborated in the inset, where the growth of the density of spreaders as time goes on is shown for the same values of the parameter $\alpha$. While the peaks of the curves get larger and larger, the times at which the maxima are reached are of the same order of magnitude and thus the meantimes of the spreading processes do not differ significantly.

Figure 2 shows another aspect worth taking into ac- count when dealing with rumor algorithms. For a given level of reliability, it is also of interest to know the distribution of ignorants (or stiflers) by classes $k$. The figure shows a coarse-grained picture of Fig. 1 where the density of ignorants $i_{k}$ according to the connectivity of the individuals has been represented for different values of $\alpha$. The results indicate that the probability of having an ignorant with a connectivity $k$, at the end of the rumor propagation, decays exponentially fast with a sharp cutoff $k_{c}$ for large connectivity values, which depends on $\alpha$. In fact, $k_{c}$ is always well below the natural cut-off of the connectivity distribution $\left(\sim 10^{2}\right)$ even for small values of $\alpha$. This implies that hubs effectively learn the rumor.

We can further scrutinize the dynamics of the rumor spreading process by looking at the final density of stiflers when the initial spreader has a given connectivity $k_{i}$. Figure 3 represents the reliability as a function of time (in units of $\alpha^{-1}$ ) when the rumor starts propagating from a node of connectivity $k_{i}=k_{\min }=3, k_{i}=\langle k\rangle=6$, $k_{i}=20$ and $k_{i}=k_{\max } \sim 280$ for two different values of $\alpha: 0.1$ (main figure) and 1.0 (inset). Interestingly, the final value of $R(t)$ does not depend on the initial seed, but reaches the same level irrespective of the connectivity of the very first spreader $k_{i}$. This is a genuine behavior of the rumor dynamics and is the opposite to what has been observed in other epidemic models like the SIR model [8], where the final number of recovered individuals strongly depends on the connectivity of the initially infected individuals. However, a closer look to the spreading dynamics tell us that not all is the same for different initial spreaders.

The figure also indicates that as the connectivity of the seed is increased, the time it takes for the rumor to reach the asymptotic value decreases, so that for a fixed time length the number of individuals in the stifler class is higher when $k_{i}$ gets larger. This feature suggests an interesting alternative for practical applications: start propagating the rumor from the most connected nodes. Even in case that no direct link exits between a node that is willing to spread an update and a hub, a dynamical (or temporal) shortcut to a well connected node could be created in order to speed up the process. With this procedure, the density of stiflers at the intermediate stages of the spreading process could be as much different as $30 \%$ for moderate values of $\alpha$. This translates in less costs, because one can always implement an algorithm that kill off the actual spreading when a given level of reliability is reached. Note, however, that this behavior slightly depends on $\alpha$, being the differences always appreciable, but more important as $\alpha$ increases.

Finally, we have exploited the fastness of the stochastic approach used here to explore the consequences of implementing three different annihilation rules for the rumor spreading decay. In particular, we consider that the spreading process dies out proportionally only to the number of spreaders ( $s s$ interactions) or to the number of stiflers ( $s r$ interactions). This modifies the terms entering in the sum of Eqs. (10 11) so that now the transition 


\begin{tabular}{|c|c|c|c|}
\hline \hline$\alpha$ & $R^{s(s+r)}$ & $R^{s r}$ & $R^{s s}$ \\
\hline 1 & 0.592 & 0.857 & 0.985 \\
0.9 & 0.635 & 0.886 & 0.989 \\
0.8 & 0.674 & 0.911 & 0.991 \\
0.7 & 0.710 & 0.938 & 0.993 \\
0.6 & 0.766 & 0.960 & 0.993 \\
0.5 & 0.818 & 0.967 & 0.997 \\
0.4 & 0.871 & 0.980 & 0.997 \\
0.3 & 0.925 & 0.997 & 0.998 \\
0.2 & 0.962 & 0.999 & 0.999 \\
0.1 & 0.988 & 0.999 & 0.999 \\
\hline \hline
\end{tabular}

TABLE II: Density of stiflers at the end of the rumor spreading process. Results are shown for 10 different values of $\alpha$ for each annihilation term considered. Simulations were performed for a network with $\langle k\rangle=6$ and $N=10^{4}$ nodes. See the text for further details.

probabilities from the $s$ into the $r$ class read

$$
\begin{aligned}
& W_{s \rightarrow r}^{s s}(t, k)=\alpha k N P(k) s_{k}(t) \sum_{k^{\prime}} \frac{k^{\prime} P\left(k^{\prime}\right) s_{k^{\prime}}(t)}{\langle k\rangle} \\
& W_{s \rightarrow r}^{s r}(t, k)=\alpha k N P(k) s_{k}(t) \sum_{k^{\prime}} \frac{k^{\prime} P\left(k^{\prime}\right) r_{k^{\prime}}(t)}{\langle k\rangle}
\end{aligned}
$$

respectively. Table II summarizes the reliability of the process as a function of $\alpha$ for the three mechanisms considered [33]. The results indicate that in all variants, the final density of stifler individuals is higher than for the "classical" setting. However, in order to evaluate the efficiency of the process from a global perspective, we must look at the time evolution of the densities as we did before.

In Figs. 4 and 5 we have represented the time (in units of $\alpha^{-1}$ ) profiles of $R(t)$ and $S(t)$ for each decay term and several values of $\alpha$. From the figures, it is clear that while the final density of stiflers increases when modifying the original decay rules, the time needed to reach such high levels of reliability also increases. This is due to the fact that the tails of the densities of spreaders decay more slowly than before. In particular, it is noticeable that when only spreader-spreader interactions are taken into account in the decay mechanism, the lifetime of the propagation process is more than two times longer than for the other two settings. This means that this implementation is not very suitable for practical applications as the costs associated to the process rise as well. On the other hand, the performance of the spreader-stifler setting seems to depend on the value of $\alpha$ in such a way that it is more efficient at both the reliability level and time consumption for a large $\alpha$, but not in the middle region of the parameter space. In summary, the present results support that the original model works quite well under any condition, while other variants can be considered depending on the value of $\alpha$ used and the type of

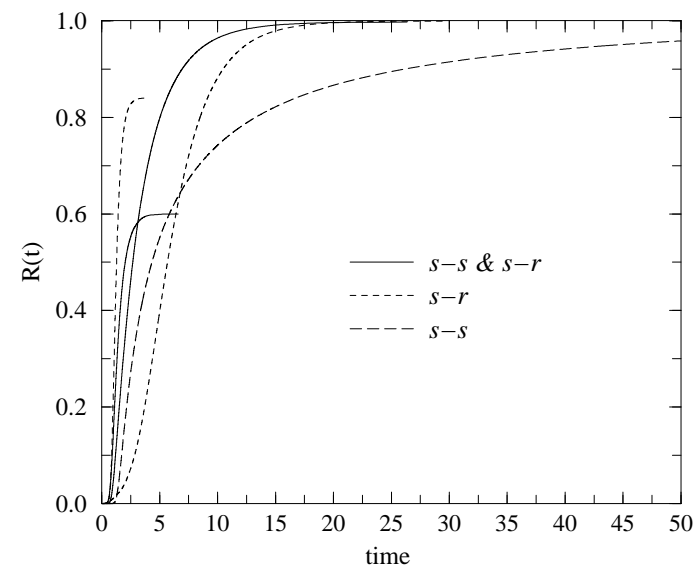

FIG. 4: Stifler's growth as a function of time for three different annihilation mechanisms as explained in the text. Curves show the time profiles for the two extreme values of $\alpha$ used in the simulations: $\alpha=1.0$ (lower curves) and 0.1 (higher curves). The curve for $s-s$ interactions is for $\alpha=0.1$ and is not complete for clarity. Time is in units of $\alpha^{-1}$.

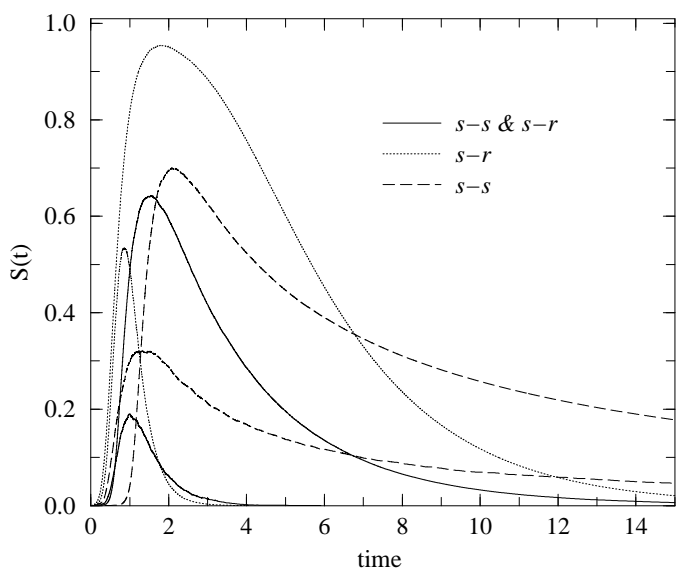

FIG. 5: Growth and decay of the populations of spreaders when the annihilation mechanism includes interactions of the type $s-s$ and $s-r$ or only $s-r$ or $s-s$. Curves show the time profiles for the two extreme values of $\alpha$ used in the simulations: $\alpha=1.0$ (lower curves) and 0.1 (higher curves). Note that although the final number of stiflers when only $s-s$ interactions enter in the decay mechanism, the time it takes for the rumor to reach the asymptotic value is very high as compared to the other two mechanisms. Curves corresponding to the $s-s$ interactions are not complete for clarity. Time is in units of $\alpha^{-1}$.

applications they are designed for.

\section{CONCLUSIONS}

In this paper, we have analyzed the spreading dynamics of rumor models in complex heterogeneous networks. 
We have first introduced a useful stochastic method that allows us to obtain meaningful time profiles for the quantities characterizing the propagation process. The method is based on the numerical solution of the meanfield rate equations describing the model, and contrary to Monte Carlo simulations, there is no need of generating explicitly the network. This allows to save memory and a fast exploration of the whole evolution diagram of the process.

The kind of processes studied here are of great practical importance since epidemic data dissemination might become the standard practice in multiple technological applications. The results show that there is a fragile balance between different levels of reliability and the costs (in terms of time) associated to them. In this sense, our study may open new paths in the use of rumormongering process for replicated database maintenance, reliable group communication and peer to peer networks [15, 16, 17, 18, 19, 20]. Besides, as shown here, the behavior and features of the different algorithms one may implement are not trivial and depend on the type of mechanisms used for both the creation and the annihilation terms. It is worth noting here that we have studied the simplest possible set of rumor algorithms, but other ingredients such as memory must be incorporated in more elaborated models [29, 30].
Of further interest would be a more careful exploration of the possibility of using dynamical shortcuts for a more efficient spreading of the updates. Our results suggest that it would be more economic to start from hubs and then kill off the updating process when a given level of reliability is reached than starting at random and letting the process dies out by itself. Preliminary studies of more elaborated models aimed at implementing a practical protocol confirm our results 30]. This feature is specially relevant for the understanding and modeling of social phenomena such as the spreading of new ideas or the design of efficient marketing campaigns.

\section{Acknowledgments}

We would like to thank A. Vespignani for many useful discussions and comments. Y. M. thanks M. VázquezPrada for helpful comments and the hospitality of BT Exact, U.K. where parts of this work were carried out. Y. M. acknowledges financial support from the Secretaría de Estado de Educación y Universidades (Spain, SB20000357). This work has been partially supported by the Spanish DGICYT project BFM2002-01798.
[1] S. N. Dorogovtsev and J. F. F. Mendes, Adv. Phys. 51, 1079 (2002).

[2] R. Albert and A.-L. Barabási, Rev. Mod. Phys. 74, 47 (2002).

[3] S. H. Strogatz, Nature (London) 410, 268 (2001).

[4] D. J. Watts and H. S. Strogatz, Nature 393, 440 (1998).

[5] L. A. N. Amaral, A. Scala, M. Barthélémi, and H. E. Stanley, Proc. Nat. Acad. Sci. 97, 11149 (2000).

[6] A.-L. Barabási, and R. Albert, Science 286, 509 (1999); A.-L. Barabási, R. Albert, and H. Jeong, Physica A 272, 173 (1999).

[7] R. Pastor-Satorras, and A. Vespignani, Phys. Rev. Lett. 86, 3200 (2001).

[8] Y. Moreno, R. Pastor-Satorras, and A. Vespignani, Eur. Phys. J. B 26, 521 (2002).

[9] R. Pastor-Satorras, and A. Vespignani, Handbook of Graphs and Networks: From the Genome to the Internet, eds. S. Bornholdt and H.G. Schuster (Wiley-VCH, Berlin, 2002).

[10] A. Vázquez, and Y. Moreno, Phys. Rev. E 67, 015101(R) (2003).

[11] M. E. J. Newman, Phys. Rev. E 66, 016128 (2002).

[12] R. Cohen, K. Erez, D. ben-Avraham, and S. Havlin, Phys. Rev. Lett. 85, 4626 (2000).

[13] D. S. Callaway, M. E. J. Newman, S. H. Strogatz, and D. J. Watts, Phys. Rev. Lett. 85, 5468 (2000).

[14] R. Albert, H. Jeong, and A.-L. Barabási, Nature 406, 378 (2000).

[15] Dave Kosiur, "IP Multicasting: The Complete Guide to Interactive Corporate Networks", Wiley Computer Publishing, John Wiley \& Sons, Inc, New York (1998).
[16] S. Deering, "Multicast routing in internetworks and extended LANs", in Proc. ACM Sigcom '88, page 55-64, Stanford, CA, USA (1988).

[17] Vogels, W., van Renesse, R. and Birman, K., "The Power of Epidemics: Robust Communication for Large-Scale Distributed Systems", in the Proceedings of HotNets-I, Princeton, NJ (2002).

[18] A.-M. Kermarrec, A Ganesh and L. Massoulie, "Probabilistic reliable dissemination in large-scale systems", IEEE Trans. Parall. Distr. Syst., in press (2003).

[19] Peer-to-Peer: Harnessing the Power of Disruptive Technologies, ed. A. Oram (O'Reilly \& Associates, Inc., Sebastopol, CA, 2001).

[20] A. J. Demers, D. H. Greene, C. Hauser, W. Irish, and J. Larson, Epidemic Algorithms for Replicated Database Maintenance. In Proc. of the Sixth Annual ACM Symposium on Principles of Distributed Computing, Vancouver, Canada, 1987.

[21] I. Foster and C. Kesselman, eds., The Grid: Blueprint for a Future Computing Infrastructure, Morgan Kaufman, San Francisco (1999).

[22] D. H. Zanette, Phys. Rev. E 64, 050901(R) (2001).

[23] Z. Liu, Y.-C. Lai, and N. Ye, Phys. Rev. E 67, 031911 (2003).

[24] D. J. Daley and J. Gani, Epidemic Modeling (Cambridge University Press, Cambridge UK, 2000).

[25] D. J. Daley, and D. G. Kendall, Nature 204, 1118 (1964).

[26] J. B. Gómez, Y. Moreno, and A. F. Pacheco, Phys. Rev. E 58, 1528 (1998).

[27] Y. Moreno, A. M. Correig, J. B. Gómez, and A. F. Pacheco, J. Geophys. Res. B 106, 6609 (2001). 
[28] Y. Moreno, J. B. Gómez, and A. F. Pacheco, Phys. Rev. E 68, 035103(R) (2003).

[29] Y. Moreno, M. Nekovee, and A. Vespignani, cond-mat/0311212 (2003).

[30] M. Nekovee and Y. Moreno, in preparation.

[31] A. Vazquez and M. Weigt, Phys. Rev. E 67, 027101 (2003).
[32] Y. Moreno, A. Vázquez, Europhy. Lett. 57, 765 (2002).

[33] Note that for the $s r$ variant, the initial conditions should include the existence of at least one stifler. This has been taken into account by randomly selecting a node among all the remaining ignorants. Hence the initial conditions are now $I(0)=N-2, S(0)=1$, and $R(0)=1$. 